\title{
Fluoropolymer indium-tin-oxide buffer layers for improved power conversion in organic photovoltaics
}

\author{
Bonan Kang, ${ }^{1,2, a)}$ L. W. Tan, ${ }^{1}$ and S. R. P. Silva ${ }^{1, a)}$ \\ ${ }^{1}$ Nanoelectronics Centre, Advanced Technology Institute, University of Surrey, Guildford, Surrey GU2 7XH, \\ United Kingdom \\ ${ }^{2}$ State Key Laboratory on Integrated Optoelectronics, Jilin University Region, Changchun 130012, \\ People's Republic of China
}

(Received 6 August 2008; accepted 20 August 2008; published online 29 September 2008)

\begin{abstract}
We investigate the effects of polytetrafluoroethylene (PTFE) on poly(3-hexylthiophene):phenylC61-butyric acid methyl ester (P3HT:PCBM) based organic photovoltaic (OPV) devices by inserting thermally evaporated PTFE films between indium-tin-oxide (ITO) and P3HT:PCBM layers. Significant improvement in terms of open-circuit voltage, short-circuit current, and thereby in its commensurate power conversion efficiency is achieved compared to devices with poly(3,4-ethylenedioxythiophene):poly(styrene-sulfonate) layers. The OPVs performance enhancement is attributed to the formation of an artificial dipole layer resulting from the rich, negatively charged fluorine that facilitates the hole extraction process. This result shows the high potential of PTFE as a low cost and stable ITO buffer layer for OPV devices. (C) 2008 American Institute of Physics. [DOI: 10.1063/1.2983742]
\end{abstract}

Recently, organic photovoltaics (OPVs) have emerged as promising alternatives to silicon based solar cells due to their advantages in low-cost, large-area, and mechanically flexible substrates that allow for building integrated material manufacture. $^{1-3}$ The performance of OPVs has increased substantially in the last several years. ${ }^{3,4}$ In polymer bulkheterojunction $(\mathrm{BHJ})$ organic photovoltaics, an important factor in determining the device performance is the charge transport in the active layer. Efficient charge transport could be realized by electrons and holes moving in individual pathways along an interpenetrating network to the appropriate electrodes, resulting in an external photocurrent density. As a result, the photocurrent does not solely depend on the characteristics of the photogenerated charge carriers, but also depends on the interface between the active layer and electrodes. Generally, to obtain good interfacial properties between the active layer and anode, anode interfacial layers such as poly(3,4-ethylenedioxythiophene):poly(styrenesulfonate) (PEDOT:PSS) are commonly used in OPV to enhance hole extraction and increase open-circuit voltage $\left(V_{\mathrm{oc}}\right)$, as well as enhance the large area electrical uniformity.

Even though PEDOT:PSS exhibits many advantageous properties such as good thermal stability ${ }^{5}$ in its oxidized state (doped form), high electrical conductivity, ${ }^{6}$ and excellent film formability, ${ }^{7}$ nevertheless, PEDOT:PSS applied as an inhomogeneous aqueous dispersion to OPV anodes [e.g., indium tin oxide $(\mathrm{ITO})]$ is highly acidic $(p \mathrm{H} \sim 1)$. Therefore, it is known to corrode the ITO, in particular, at elevated temperatures. Furthermore, typical spin-cast PEDOT:PSS films have large microstructural and electrical inhomogeneities, with morphology and conductivity varying by orders of magnitude over different film regions. ${ }^{8,9}$ These effects doubtless lead to inhomogeneous charge extraction with enhanced conductivity in some locations and "dead spots" in others. These issues illustrate the need for an anode interfacial layer

\footnotetext{
a) Authors to whom correspondence should be addressed. Electronic addresses: 1mkbn@mail.tsinghua.edu.cn and s.silva@surrey.ac.uk.
}

that has greater charge-blocking characteristics, has strong adhesion to the anode surface, and allows for subsequent active layers to be spin coated with greater thermal stability for the rapid commercialization of OPVs.

In this study, we report on using fluoropolymer based material called polytetrafluoroethylene (PTFE), which is better known as Teflon, as the ITO buffer layer for OPV applications. The great advantage of PTFE is its low chemical reactivity, ${ }^{10}$ thermal stability (up to $420^{\circ} \mathrm{C}$ ), ${ }^{11}$ moisture resistance, flexible, light weight, and low cost. The effect of the PTFE layer thickness upon the device efficiency has been investigated.

The device consists of ITO-coated glass substrate, PTFE layer, poly(3-hexylthiophene):phenyl-C61-butyric acid methyl ester (P3HT:PCBM), and Al cathode. The ITO glass is precleaned following an oxygen plasma treatment for $3 \mathrm{~min}$ prior to use for device fabrication. P3HT (Rieke Metals, used as received) was dissolved in 1,2-dichlorobenzene to make $17 \mathrm{mg} / \mathrm{ml}$ solution, followed by blending with PCBM (Nano-C, used as received) in 1:1 weight ratio. The blend was stirred for $\sim 14 \mathrm{~h}$ in a glovebox before being spin

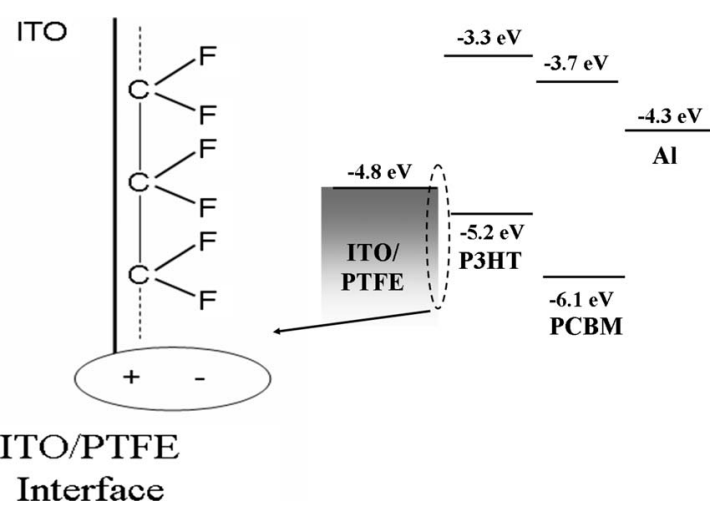

FIG. 1. Energy-level diagram shows the highest occupied molecular orbital and lowest unoccupied molecular orbital of each material in the OPV device. Inset: Schematic of the ITO/PTFE interface. 


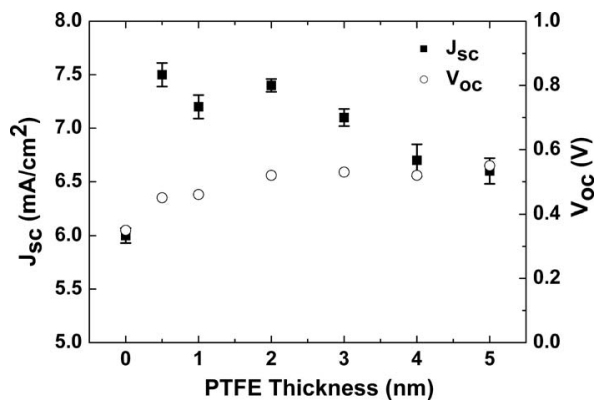

FIG. 2. Plots of the $J_{\text {sc }}$ (mean and $95 \%$ confidence standard error range) and $V_{\text {oc }}$ of P3HT: PCBM based OPV devices with different PTFE thicknesses.

coated on top of the ITO/PTFE surface. The typical film thickness of P3HT: PCBM is about $\sim 210-230 \mathrm{~nm}$. Five types of OPVs are prepared with a PTFE layer varied between 0.5 and $5.0 \mathrm{~nm}$. Both PTFE and Al films are deposited at a base pressure of $2 \times 10^{-5}$ Torr. All the measurements are carried out in ambient atmosphere at room temperature. To study the consistency of the device performance, a minimum of five samples for each individual structure of the devices are tested.

Figure 1 shows the energy levels of the single components of the photovoltaic cell with a simple schematic of the ITO/PTFE interface. The fluorine component aligns on top of the ITO surface as shown in Fig. 1, creating a dipole surface with the dipole moment directed inward the ITO. This reduces the energy barrier between the ITO and organic interface. $^{12}$

In Fig. 2, the $J_{\mathrm{sc}}$ of the OPV devices are plotted as a function of the PTFE thicknesses. Compared to the device without the PTFE buffer layer, all the devices with PTFE buffer layer showed a significant improvement by showing higher $J_{\mathrm{sc}}$. The high $J_{\mathrm{sc}}$ in OPV devices with the PTFE layer are mainly due to the reduction in the barrier height between ITO and organic matrix and subsequently improve the hole extraction process. In general, for organic electronic material systems (e.g., P3HT/PCBM), the hole mobility is a few magnitudes higher than the measured electron mobility. ${ }^{13}$ Hence, hole accumulation occurs in the device and the photocurrent is under space-charge limited conditions, ${ }^{14}$ which result in nonuniformity in the applied field within the active area or bulk of the device structure. By utilizing the PTFE buffer layer, efficient hole extraction can reduce the hole accumulation at the ITO/organic interface and as a result, the shortcircuit current $J_{\mathrm{sc}}$ is improved. Since PTFE is an insulating material, with an extremely high resistivity of $10^{18} \Omega / \mathrm{cm}$

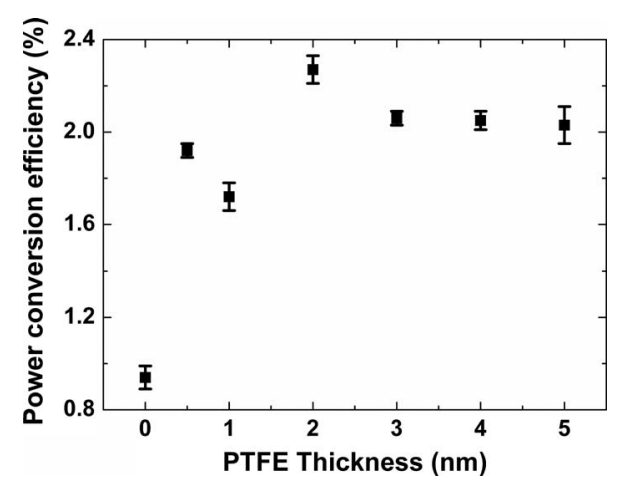

FIG. 3. PCE response plots of OPV devices with different PTFE thicknesses.

for its bulk properties and a large value for its ionization potential of $9.8 \mathrm{eV},{ }^{15}$ it is reasonable to expect that the shortcircuit current densities of OPVs decrease with increasing thickness of the PTFE layer.

As shown in Fig. 2, the $V_{\text {oc }}$ of the devices is strongly dependent on the PTFE thicknesses. For the reference devices with the structure of ITO/P3HT:PCBM/Al and ITO/ PEDOT/P3HT: PCBM, the typical $V_{\text {oc }}$ was found to be 0.35 and $0.44 \mathrm{~V}$, respectively (Table I). However, the $V_{\mathrm{oc}}$ is found to be improved up to $0.55 \mathrm{~V}$ for the device with $5 \mathrm{~nm}$ of PTFE buffer layer. The work function of the ITO surface typically varies from 3.90 to $4.80 \mathrm{eV}$ depending on the surface treatment. ${ }^{16,17}$ After inserting the PTFE layer, the $V_{\text {oc }}$ is improved from 0.35 to $0.56 \mathrm{~V}$ because of the strong dipole layer created by the negatively charged fluorine rich PTFE layer that has modified the surface and increased the effective work function of the ITO.

The improvement of $J_{\mathrm{sc}}$ and $V_{\mathrm{oc}}$ of the devices with the PTFE is mirrored in power conversion efficiency of the device shown in Fig. 3 as a function of the PTFE film thickness. As shown in Table I, the devices with PTFE buffer layer exhibited at least a $40 \%$ increase in terms of power conversion efficiency compared to the reference devices. Among the device with PTFE, a device with a $2 \mathrm{~nm}$ PTFE buffer layer exhibited the best performance with a power conversion efficiency (PCE) of $2.2 \%$, which is attributed to the good coverage of $2 \mathrm{~nm}$ PTFE layer on the ITO to modify the ITO surface without an increase in the surface resistivity of ITO/PTFE anode. This is assumed to be associated with the enhanced tunneling of charge carriers across the PTFE, help by the strong dipole layer.

TABLE I. List of device performance of OPV devices with and without different PTFE thicknesses.

\begin{tabular}{lccccc}
\hline \hline Device structure & $\begin{array}{c}\text { PTFE } \\
\text { thickness } \\
(\mathrm{nm})\end{array}$ & $\begin{array}{c}J_{\mathrm{sc}} \\
\left(\mathrm{mA} / \mathrm{cm}^{2}\right)\end{array}$ & $\begin{array}{c}V_{\mathrm{oc}} \\
(\mathrm{V})\end{array}$ & $\begin{array}{c}\mathrm{FF} \\
(\%)\end{array}$ & $\begin{array}{c}\eta \\
(\%)\end{array}$ \\
\hline ITO/PTFE/P3HT:PCBM/Al & 5.0 & 6.6 & 0.55 & 46.0 & 2.03 \\
& 4.0 & 6.7 & 0.52 & 48.6 & 2.05 \\
& 3.0 & 7.1 & 0.53 & 45.0 & 2.06 \\
& 2.0 & 7.4 & 0.52 & 48.5 & 2.27 \\
ITO/PEDOT:PSS/P3HT:PCBM/Al & 1.0 & 7.2 & 0.46 & 42.9 & 1.72 \\
ITO/P3HT:PCBM/Al & 0.5 & 7.5 & 0.45 & 47.2 & 1.92 \\
\hline \hline
\end{tabular}


In summary, insertion of the PTFE buffer layer at the anode of the ITO/organic interface can significantly improve the $J_{\mathrm{sc}}, V_{\mathrm{oc}}$, and PCE of BHJ OPV. The performance improvement is attributed to the reduction in hole extraction barrier. This fluoromaterial also offers a significant advantage in that the film can be simply prepared by thermal evaporation. Thus, it can be prepared at low substrate temperatures, which are compatible to flexible polymer substrates for OPV applications. In the future, low cost fluoropolymer (PTFE) may be used as an alternative replacement material for PEDOT:PSS.

The authors are grateful to funding from the EPSRC in the form of a Portfolio Partnership Award and China Scholarship Council.

${ }^{1}$ W. U. Huynh, J. J. Dittmer, and A. P. Alivisatos, Science 295, 2425 (2002).

${ }^{2}$ W. Ma, C. Yang, X. Gong, K. Lee, and A. J. Heeger, Adv. Funct. Mater. 15, 1617 (2005).

${ }^{3}$ G. Li, V. Shrotriya, J. Huang, Y. Yao, T. Moriarty, K. Emery, and Y. Yang, Nat. Mater. 4, 864 (2005).
${ }^{4}$ J. Y. Kim, K. Lee, N. E. Coates, D. Moses, T. Q. Nguyen, M. Dante, and A. J. Heeger, Science 317, 222 (2007).

${ }^{5}$ J. C. Gustaffson, B. Liedberg, and O. Inganas, Solid State Ionics 69, 145 (1994).

${ }^{6}$ L. Groenendaal, G. Zotti, and F. Jonas, Synth. Met. 118, 105 (2001).

${ }^{7}$ H. W. Heuer, R. W. Wehrmann, and S. Kirchmeyer, Adv. Funct. Mater. 12, 89 (2002).

${ }^{8}$ A. W. Hains and T. J. Marks, Appl. Phys. Lett. 92, 023504 (2008).

${ }^{9}$ M. Kemerink, S. Timpanaro, M. M. de Kok, E. A. Meulenkamp, and F. J. Touwslager, J. Phys. Chem. B 108, 18820 (2004).

${ }^{10}$ H. Agren, V. Carravetta, O. Vahtras, and L. G. M. Pettersson, Phys. Rev. B 51, 17848 (1995).

${ }^{11}$ L. M. Han and R. B. Timmons, J. Vac. Sci. Technol. B 18, 799 (2000).

${ }^{12}$ S. W. Tong, K. M. Lau, H. Y. Sun, M. K. Fung, C. S. Lee, Y. Lifshitz, and S. T. Lee, Appl. Surf. Sci. 252, 3806 (2006).

${ }^{13}$ H. Sirringhaus, Adv. Mater. (Weinheim, Ger.) 17, 2411 (2005).

${ }^{14}$ V. D. Mihailetchi, J. Wildeman, and P. W. M. Blom, Phys. Rev. Lett. 94, 126602 (2005).

${ }^{15}$ Y. Gao, L. Wang, D. Zhang, L. Duan, G. Dong, and Y. Qiu, Appl. Phys. Lett. 82, 155 (2003).

${ }^{16}$ F. Nüesch, L. J. Rothberg, E. W. Forsythe, Q. T. Le, and G. Yongli, Appl. Phys. Lett. 74, 880 (1999).

${ }^{17}$ J. S. Kim, M. Granström, R. H. Friend, N. Johansson, W. R. Salaneck, R. Daik, W. J. Feast, and F. Cacialli, J. Appl. Phys. 84, 6859 (1998). 